\title{
Electroweak baryogenesis and dark matter via a pseudoscalar vs. scalar
}

\author{
Parsa Hossein Ghorbani \\ Institute for Research in Fundamental Sciences (IPM), \\ School of Particles and Accelerators, \\ P.O. Box 19395-5531, Tehran, Iran \\ E-mail: pghorbani@ipm.ir
}

ABSTRACT: We study the electroweak baryogenesis in a fermionic dark matter scenario with a (pseudo)scalar being the mediator in the Higgs portal. It is discussed that the electroweak phase transition turns to be first-order after taking into account the role of the (pseudo)scalar in the thermal effective potential in our extended standard model. Imposing the relic density constraint from the WMAP/Planck and the bounds from the direct detection experiments XENON100/LUX, we show that the dark matter scenario with a scalar mediator is hardly capable of explaining the baryogenesis while the same model with a pseudoscalar mediator is able to explain the baryon asymmetry. For the latter, we constrain more the model with Fermi-LAT upper limit on dark matter annihilation into $b \bar{b}$ and $\tau^{+} \tau^{-}$. The allowed dark matter mass that leads to correct relic abundance, renders the electroweak phase transition strongly first-order, and respects the Fermi-LAT limit, will be in the range 110-320 GeV. The exotic and invisible Higgs decay bounds and the mono-jet search limit at the LHC do not affect the viable space of parameters.

KeYwords: Beyond Standard Model, Cosmology of Theories beyond the SM

ARXIV EPRINT: 1703.06506 


\section{Contents}

1 Introduction 1

2 The model 2

3 First-order phase transition 3

4 Fermionic dark matter 5

$\begin{array}{lll}5 & \text { Numerical results } & 6\end{array}$

6 Conclusion $\quad 9$

A (Pseudo)scalar one-loop mass correction by dark matter fermion $\quad 10$

\section{Introduction}

The electroweak symmetry breaking which we believe is a cornerstone in the Higgs physics could be restored at high temperature i.e. in the early universe [1, 2]. The spontaneous symmetry breaking occurs by having the universe expanded and cooled and going from the symmetric phase to the broken phase through the electroweak phase transition (EWPT).

An open problem in physics is the mysterious matter-antimatter asymmetry in the universe. The standard model (SM) together with the EWPT seem to be able to explain the exceed of the matter abundance against the antimatter we observe today. This is called the electroweak baryogenesis (EWBG). The necessary conditions that lead to EWBG is known as the three criteria of Sakharov [3]: Baryon number violation, $\mathrm{C}$ and $\mathrm{CP}$ violation and the thermal non-equilibrium. The standard model possesses all these conditions however at the temperature of the electroweak symmetry breaking, $T_{\mathrm{EW}} \sim 100 \mathrm{GeV}$, the electroweak phase transition must be of first-order type in order to support the EWBG. This can be translated into washout criterion, $v\left(T_{c}\right) / T_{c}>1$ which provides the appropriate sphaleron rate for the baryogenesis. In the standard model framework the washout condition impose an upper limit on the Higgs mass: $m_{H}<48 \mathrm{GeV}$ [4] which is in contrast with the Higgs discovery $\left(m_{H} \sim 125 \mathrm{GeV}\right)$ at the LHC in $2012[5,6]$. Therefore the SM alone is not enough to embed the EWBG and an extension to SM looks necessary if the electroweak baryogenesis is the right mechanism to produce the baryon asymmetry.

On the other hand, almost $25 \%$ of the universe is made of what we call it dark matter (DM). It does not have strong interaction with the ordinary matter and its existence can be traced only through its gravitational attraction on matter [7, 8]. Again the SM is not capable of explaining this extra gravitational force in the galaxies and clusters. Inevitably one should search for an answer in a theory beyond the standard model. One 
of the successful DM scenarios is the weakly interacting massive particle (WIMP) which demands additional fundamental fields (particles) respect to the SM. The new particle(s) stay in thermal equilibrium with other SM particles in the early universe, but when the universe cools down while expanding, it freezes-out from the hot plasma of particles. The self-annihilation cross section of the WIMP must be of order $\langle\sigma v\rangle=3 \times 10^{-26} \mathrm{~cm}^{3} \mathrm{~s}^{-1}$ to have the correct amount of DM abundance to be $\Omega h^{2} \sim 0.11$ measured by Planck/WMAP.

In this work we try to address both the electroweak baryogenesis and the dark matter issues in one single theory. We extend the standard model by adding an extra Dirac fermion playing the role of the dark matter candidate and a (pseudo)scalar mediating between the dark sector and the SM sector [9]. ${ }^{1}$ The (pseudo)scalar and the fermionic dark matter modify the thermal effective potential; hence affect the critical temperature for the electroweak phase transition. We analytically provide the critical temperature and the global minimum at the critical temperature to give the washout criterion. Many works have provided the critical temperature from the free energy by numerical computation. Some examples of the works done considering both the dark matter and the baryogenesis are [11-20]. Here we provide an analytical expression for the critical temperature which makes it easier to impose the washout condition on the dark matter model.

If the SM-DM mediator is a pseudoscalar the dark matter cross section off nucleon is negligible while if the mediator is a scalar then the direct detection experiments e.g. XENON100/LUX put a strong constraint on the space parameter. Having emphasized this point, we show in this work that the dark matter model with a pseudoscalar mediator is more successful in explaining the electroweak baryogenesis respect to when we use the scalar mediator.

This paper is organized as the following: in the next section we introduce the model by extending the SM. In section 3 the details of the electroweak phase transition and the critical temperature are given. In section 4 , the dark matter candidate is introduced. In the next section, the numerical results that shows the consistency of the model with relic density and the baryon asymmetry is presented. The appendix A provides some analytical details on the effective potential.

\section{The model}

We extend the standard model by adding two new fields as follows: a Dirac fermion denoted here by $\psi$, which plays the role of the dark matter candidate, and a (pseudo)scalar denoted by $s$ which mixes with the Higgs field, $H$, and interacts with the dark matter as well through a Yukawa term. The Lagrangian can be written in its parts as,

$$
\mathcal{L}=\mathcal{L}_{\mathrm{SM}}(H)+\mathcal{L}_{\text {dark }}(\psi)+\mathcal{L}_{s}(s)+\mathcal{L}_{\text {int }}(s, \psi, H),
$$

where $\mathcal{L}_{\mathrm{SM}}$ stands for the SM Lagrangian, $\mathcal{L}_{\text {dark }}$ for the fermionic dark matter Lagrangian,

$$
\mathcal{L}_{\text {dark }}(s, \psi)=\bar{\psi}\left(i \gamma_{\mu} \partial^{\mu}-m_{d}\right) \psi,
$$

\footnotetext{
${ }^{1}$ See [10] for the analysis of the renormalization group equation for the same model with a pseudoscalar mediator.
} 
$\mathcal{L}_{s}$ for the (pseudo)scalar Lagrangian,

$$
\mathcal{L}_{s}=\frac{1}{2}\left(\partial_{\mu} s\right)^{2}-\frac{1}{2} \mu_{s}^{2} s^{2}-\frac{1}{4} \lambda_{s} s^{4},
$$

and $\mathcal{L}_{\text {int }}$ is the (pseudo)scalar interaction with the dark and the SM sectors. When $s$ is a pseudoscalar then,

$$
\mathcal{L}_{\text {int }}(s, \psi, H)=g_{d} s \bar{\psi} \gamma^{5} \psi+\frac{1}{2} \lambda s^{2} H^{\dagger} H
$$

and when $s$ represents the scalar,

$$
\mathcal{L}_{\text {int }}(s, \psi, H)=g_{d} s \bar{\psi} \psi+\frac{1}{2} \lambda s^{2} H^{\dagger} H .
$$

In the next sections we investigate both the scalar and the pseudoscalar cases and use the same notation for the coupling $g_{d}$.

Note that the interaction Lagrangian does not include the odd-terms in the (pseudo)scalar-Higgs interaction terms. In order to stay in a more restricted theory with as small parameter space as possible, despite many authors we consider a less general Lagrangian for our model. The Higgs potential in the SM sector reads,

$$
V_{H}=-\mu_{h}^{2} H^{\dagger} H-\lambda_{h}\left(H^{\dagger} H\right)^{2}
$$

Both the Higgs and the (pseudo)scalar take non-zero vacuum expectation values at the low temperature. In section 3 to give the thermal effective potential as a function of the condensate $\langle h\rangle$ we begin with the tree-level potential which is given by the substitution $s \equiv\langle s\rangle$ and $H=(0 h \equiv\langle h\rangle)^{\dagger}$ So the tree-level potential reads,

$$
V_{0}(h, s)=-\frac{1}{2} \mu_{h}^{2} h^{2}-\frac{1}{2} \mu_{s}^{2} s^{2}+\frac{1}{4} \lambda_{h} h^{4}+\frac{1}{4} \lambda_{s} s^{4}+\frac{1}{2} \lambda s^{2} h^{2} .
$$

Note that we have gauged away three degrees of freedom of the Higgs doublet.

\section{First-order phase transition}

In this section we provide the "washout criterion" and other necessary conditions in terms of the parameters used in the model introduced above to support the first-order electroweak phase transition. The washout criterion or $v\left(T_{c}\right) / T_{c}>1$ which provides the appropriate sphaleron rate for the phase transition to be first-order is obtained from the effective potential of the theory. In addition to the tree-level barrier we also consider the one-loop barrier. The total thermal effective potential is therefore,

$$
V_{\text {eff }}=V_{0}(h, s)+V_{1-\text { loop }}(h, s ; 0)+V_{1-\text { loop }}(h, s ; T),
$$

where $V_{0}$ is the tree-level potential in eq. $(2.7), V^{1-l o o p}(h, s ; 0)$ is the Coleman-Weinberg one-loop correction at zero temperature [21] and $V^{1 \text {-loop }}(h, s ; T)$ is the one-loop thermal correction [2]. In the high-temperature approximation when $m_{i}^{2} / T^{2} \ll 1$ for all $i$ with $m_{i}$ 
being the mass of the particle $i$ in the model, the one-loop effective potential takes the following form,

$$
V_{1-\text { loop }}^{\text {high-T }}(h, s ; T) \approx\left(\frac{1}{2} \kappa_{h} h^{2}+\frac{1}{2} \kappa_{s} s^{2}\right) T^{2},
$$

with

$$
\begin{aligned}
\kappa_{h} & =\frac{1}{48}\left(9 g^{2}+3 g^{2}+12 g_{t}^{2}+24 \lambda_{h}+4 \lambda\right), \\
\kappa_{s} & =\frac{1}{12}\left(4 \lambda+3 \lambda_{s} \pm 2 g_{d}^{2}\right)
\end{aligned}
$$

where in eq. (3.4) the minus sign stands for the pseudoscalar case and the plus sign is for the scalar case. See appendix A for more details.

Note that we have dropped the Colman-Wienberg zero-temperature correction since at high temperature approximation (at temperature of the electroweak phase transition) only the thermal corrections are dominant. Moreover, including the zero-temperature contribution will only complicate the analytic computations.

In eq. (3.3) the parameters $g$ and $g^{\prime}$ are respectively the $\mathrm{SU}(2)_{L}$ and $\mathrm{U}(1)_{Y}$ standard model couplings and $g_{t}$ is the top quark coupling. We have contributed only the heavier particles that couple stronger to the Higgs, i.e. the top quark, $t$, the gauge bosons, $W^{ \pm}$ and $Z$, and the Higgs, $h$. We have ignored the lighter quarks, gluons and the leptons.

In order to have a first-order phase transition the effective potential must have two degenerate minima at the critical temperature. The minima are located at

$$
\left.\frac{\partial V_{\mathrm{eff}}}{\partial s}\right|_{w(T)=<s>}=\left.0 \quad \frac{\partial V_{\mathrm{eff}}}{\partial h}\right|_{v(T)=<h>}=0,
$$

where leads to

$$
\begin{aligned}
v_{\text {sym }}=0 \quad \text { or } \quad v_{\text {brk }}^{2}(T) & =\frac{\mu_{h}^{2}-\kappa_{h} T^{2}-\lambda w^{2}(T)}{\lambda_{h}}, \\
v_{\text {brk }}^{2}(T) & =\frac{\mu_{s}^{2}-\kappa_{s} T^{2}-\lambda_{s} w^{2}(T)}{\lambda} .
\end{aligned}
$$

The stability conditions are obtained by setting the positivity of the second derivatives of the potential after the symmetry breaking:

$$
\lambda_{s}>0, \quad \lambda_{h}>0, \quad 4 \lambda_{s} \lambda_{h}>\lambda^{2} .
$$

Eqs. (3.6) and (3.7) leads to

$$
w_{\mathrm{brk}}^{2}(T)=\alpha+\beta T^{2}
$$

where

$$
\begin{aligned}
\alpha & =\frac{\lambda \mu_{h}^{2}-\lambda_{h} \mu_{s}^{2}}{\lambda^{2}-\lambda_{h} \lambda_{s}}, \\
\beta & =\frac{\lambda \kappa_{h}-\lambda_{h} \kappa_{s}}{\lambda^{2}-\lambda_{h} \lambda_{s}} .
\end{aligned}
$$


Using eq. (3.6) one gets the temperature-dependent Higgs vacuum expectation value in the broken phase,

$$
v_{\mathrm{brk}}^{2}(T)=\left(\frac{\mu_{h}^{2}-\lambda \alpha}{\lambda_{h}}\right)-\left(\frac{\kappa_{h}+\lambda \beta}{\lambda_{h}}\right) T^{2} .
$$

The broken phase can exist up to a maximal temperature,

$$
T_{\max }=\sqrt{\frac{\mu_{h}^{2}-\lambda \alpha}{\kappa_{h}+\lambda \beta}}, \quad \mu_{h}^{2}>\lambda \alpha .
$$

At the critical temperature the free energy has two degenerate minima one at $\left(v_{\mathrm{sym}}=0, w_{\mathrm{sym}}=0\right)$ and the other at $\left(v_{\mathrm{brk}}, w_{\mathrm{brk}}\right)$,

$$
V_{\mathrm{eff}}^{\mathrm{sym}}\left(T_{c}\right)=V_{\mathrm{eff}}^{\mathrm{brk}}\left(T_{c}\right)=0,
$$

which solves the free energy eq. (3.14) at the critical temperature as,

$$
T_{c}^{ \pm}=\sqrt{x \pm y^{1 / 2} / z}
$$

with

$$
\begin{aligned}
x= & \kappa_{h} \lambda_{h}\left(\lambda \mu_{s}^{2}-\lambda_{s} \mu_{h}^{2}\right)+\kappa_{s} \lambda_{h}\left(\lambda \mu_{h}^{2}-\lambda_{h} \mu_{s}^{2}\right), \\
y= & \kappa_{h}{ }^{2} \lambda_{h}^{2}\left(-8 \lambda^{3} \mu_{h}^{2} \mu_{s}^{2}+\lambda^{2}\left(5 \lambda_{h} \mu_{s}{ }^{4}+4 \lambda_{s} \mu_{h}{ }^{4}\right)-\lambda_{h}{ }^{2} \lambda_{s} \mu_{s}{ }^{4}\right) \\
& +2 \kappa_{h} \kappa_{s} \lambda_{h}^{3}\left(7 \lambda^{2} \mu_{h}^{2} \mu_{s}^{2}-4 \lambda\left(\lambda_{h} \mu_{s}{ }^{4}+\lambda_{s} \mu_{h}{ }^{4}\right)+\lambda_{h} \lambda_{s} \mu_{h}^{2} \mu_{s}^{2}\right) \\
& +\kappa_{s}{ }^{2} \lambda_{h}^{3}\left(\lambda^{2} \mu_{h}{ }^{4}-8 \lambda \lambda_{h} \mu_{h}^{2} \mu_{s}^{2}+\lambda_{h}\left(4 \lambda_{h} \mu_{s}{ }^{4}+3 \lambda_{s} \mu_{h}{ }^{4}\right)\right), \\
z= & \kappa_{h}{ }^{2}\left(4 \lambda^{2}-\lambda_{h} \lambda_{s}\right)-6 \kappa_{h} \kappa_{s} \lambda \lambda_{h}+3 \kappa_{s}{ }^{2} \lambda_{h}{ }^{2} .
\end{aligned}
$$

The strong electroweak phase transition occurs only if

$$
v\left(T_{c}\right) / T_{c}>1
$$

which is a strong constraint on the parameter space of our dark matter model. Note that for each set of the couplings in eq. (3.16) there are two critical temperatures, $T_{c}^{ \pm}$, if $x \pm y^{1 / 2} / z>0$. We will see in section 4 that for both solutions there exist a viable parameter space.

\section{Fermionic dark matter}

We are assuming that the first-order phase transition is occurring in a temperature higher than the dark matter freeze-out temperature, i.e. $T_{c}>T_{F}$. After the symmetry breaking both Higgs particle and the scalar field undergo non-zero vev:

$$
\begin{gathered}
h \rightarrow v_{h}+h, \\
s \rightarrow v_{s}+s .
\end{gathered}
$$


This will cause to a three potential different from eq. (2.7). One can redefine the scalar fields $h$ and $s$ in order to diagonalize the mass matrix as follows,

$$
\begin{aligned}
& h \rightarrow h \cos \theta+s \sin \theta, \\
& s \rightarrow-h \sin \theta+s \cos \theta .
\end{aligned}
$$

For simplicity we denote the new fields as $h$ and $s$ again but one should be noted that these are the eigenstates of the diagonal mass matrix now. Plugging eqs. (4.1) and (4.2) in eq. (2.7) and imposing the minimization condition we get

$$
\begin{aligned}
& \mu_{s}^{2}=-\lambda_{s} v_{s}^{2}-\lambda v_{h}^{2}, \\
& \mu_{h}^{2}=-\lambda_{h} v_{h}^{2}-\lambda v_{s}^{2} .
\end{aligned}
$$

The second derivative of the potential in eq. (2.7) after shifting the origin to the non-zero vacuum are,

$$
\tilde{m}_{s}^{2}=2 \lambda_{s} v_{s}^{2}, \quad \tilde{m}_{h}^{2}=2 \lambda_{h} v_{h}^{2}, \quad \tilde{m}_{h s}^{2}=\lambda v_{h} v_{s} .
$$

Diagonalizing the mass matrix by a rotation by the angle $\theta$, the couplings will be related to the masses as,

$$
\begin{aligned}
\lambda_{h} & =\frac{m_{s}^{2} \sin ^{2} \theta+m_{h}^{2} \cos ^{2} \theta}{2 v_{h}^{2}}, \\
\lambda_{s} & =\frac{m_{s}^{2} \cos ^{2} \theta+m_{h}^{2} \sin ^{2} \theta}{2 v_{s}^{2}} \\
\lambda & =\frac{m_{s}^{2}-m_{h}^{2}}{2 v_{h} v_{s}} \sin 2 \theta
\end{aligned}
$$

where $m_{s}^{2}$ and $m_{h}^{2}$ are the diagonalized masses and

$$
\tan \theta=\frac{y}{1+\sqrt{1+y^{2}}}, \quad y=\frac{2 \lambda v_{s} v_{h}}{\lambda_{h} v_{h}^{2}-\lambda_{s} v_{s}^{2}} .
$$

The independent parameters of the model can then be chosen as $m_{h}, m_{s}, m_{d}, g_{d}$ and $\sin \theta$. All the other parameters including the mass terms and the couplings in the Lagrangian are expressed in terms of these five parameters. Note that all we have said in this section is true for both scalar and pseudoscalar mediators. In the next section we see how the numerical results changes for them.

\section{Numerical results}

Our computations are two-folded. One part is the computations for the model with a pseudoscalar mediator and the other part is the computations with a scalar mediator. We recall that the difference they make in strongly first-order phase transition, section 3 , stem from eq. (3.4) where the plus sign stands for the scalar and the minus sign stands for the pseudoscalar. In dark matter side apart from the different results for the relic density for each type of mediator, one should note an important point; when choosing the mediator 

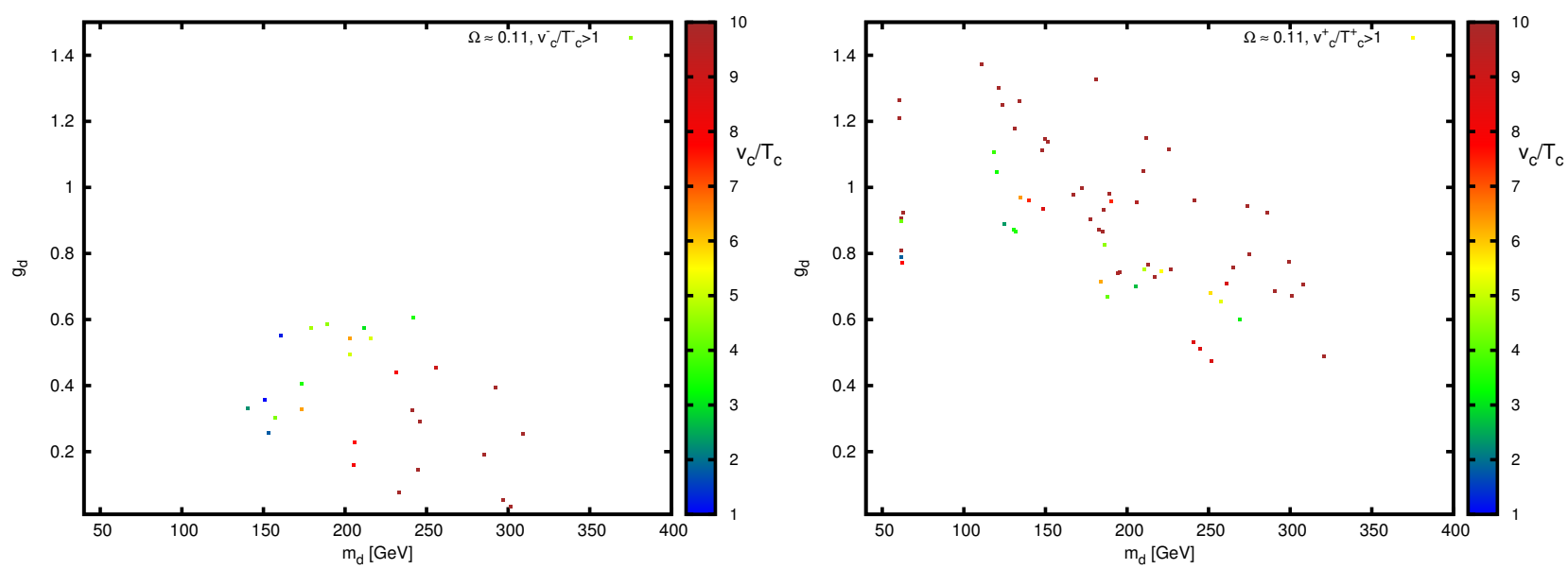

Figure 1. The allowed DM mass, $m_{d}$, against the Yukawa coupling, $g_{d}$, in the fermionic dark matter scenario with pseudoscalar mediator, bounded from the relic density and first-order phase transition conditions for left) $v_{c}^{-} / T_{c}^{-}>1$ with DM mass in the range $140-310 \mathrm{GeV}$ right) $v_{c}^{+} / T_{c}^{+}>1$ with DM mass in the range $60-320 \mathrm{GeV}$.

in our dark matter scenario to be a pseudoscalar the elastic scattering cross section for DM-nucleon is velocity suppressed $[9,22-24]$ and so the theory easily evades the direct detection bounds from LUX/XENON100/XENON1T [25-27]. However this is not the case for a scalar mediator. A large region of the parameter space in the theory is cut by the direct detection bounds imposed from XENON100/LUX. Therefore, for the pseudoscalar mediator we have two type of constraints to impose: the relic density and the first-order phase transition condition. For the scalar mediator the direct detection constraint must be added to the above conditions.

To compute the relic density for the dark matter one should solve the Boltzmann differential equation numerically. We have exploited the MicrOMEGAs4.3 package [28] to obtain the relic abundance. The Higgs vacuum expectation value and the Higgs mass are known, $v_{h}=246 \mathrm{GeV}$ and $m_{h}=125 \mathrm{GeV}$. The vev for the pseudoscalar and for the scalar as well is chosen to be $v_{s}=600 \mathrm{GeV} .^{2}$ In both cases, we search for the viable parameter space bounded by the relic density value $\Omega h^{2}=0.11$ and the washout condition being $v\left(T_{c}\right) / T_{c}>1$ with $v\left(T_{c}\right)$ and $T_{c}$ given in eqs. (3.15) and (3.12). We scan the space of parameters in the ranges as $\mathrm{GeV} 1<m_{s}<1 \mathrm{TeV}$, the dark matter mass $\mathrm{GeV} 10<m_{d}<$ $2 \mathrm{TeV}$, the Yukawa coupling $0<g_{d}<3$ and the mixing angle being fixed at $\sin \theta=0.1$.

As we have demonstrated in figures 1 and 2 that despite the strong constraints on the parameter space we end up with a viable region for the theory figure 1 shows the allowed DM mass against the Yukawa coupling for the dark matter model with the pseudoscalar mediator and figure 2 represents the same quantities for the scalar mediator. The constraints considered in two figures are the amount of the relic abundance from WMAP/Planck, the washout criterion for two critical temperature found in eq. (3.15) and the DM-nucleon elastic scattering cross section bound from XENON100/XENON1T/LUX.

\footnotetext{
${ }^{2} \mathrm{~A}$ caveat here is that additional (pseudo)scalars in the extended SM with non-zero changing vev during the electroweak phase transition may lead to supersonic bubble wall velocity that prevent the phase transition from being strong enough for EWBG [29, 30].
} 

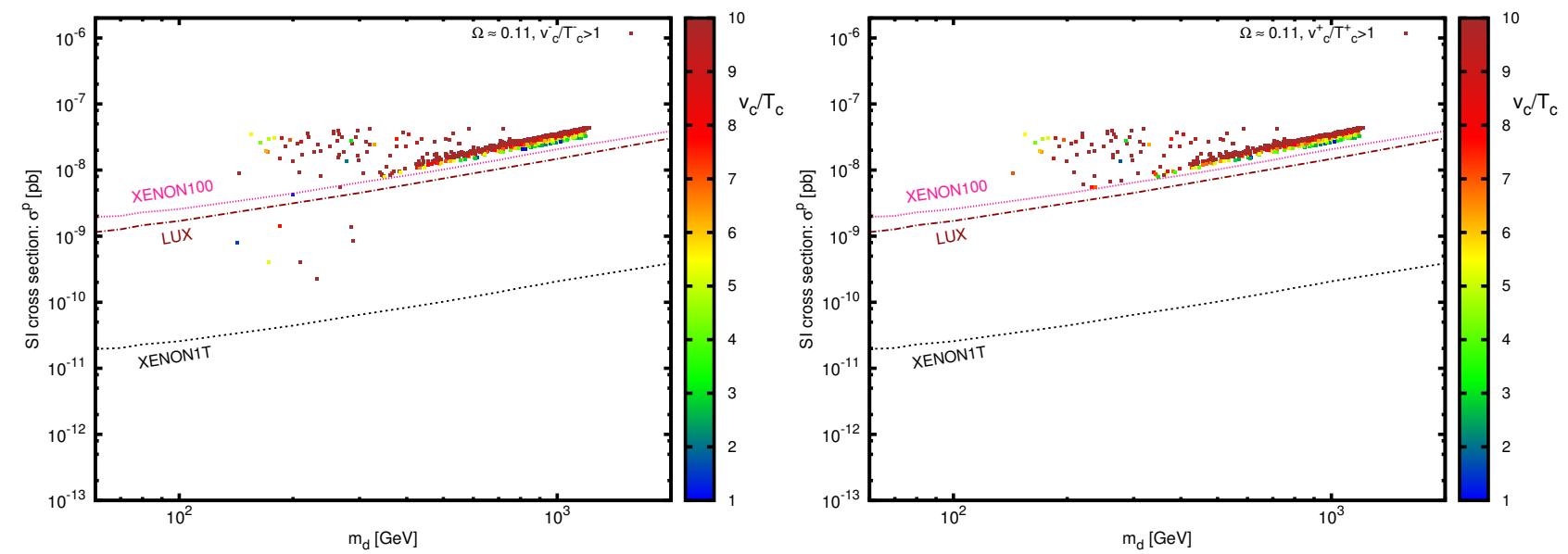

Figure 2. The allowed DM mass, $m_{d}$, against the Yukawa coupling, $g_{d}$, in the fermionic dark matter scenario with scalar mediator, bounded from the relic density, first-order phase transition condition, and the direct detection bound by XENON100/XENON1T/LUX for left) $v_{c}^{-} / T_{c}^{-}>1$ with DM mass in the range 150-300 GeV for XENON100/LUX and no viable DM mass for the future direct detection bound by XENON1T right) $v_{c}^{+} / T_{c}^{+}>1$ with no viable DM mass.

It is interesting to point out the difference in two figures; the fermionic dark matter model with a pseudoscalar mediator figure 1 is more successful respect to the same model with a scalar mediator figure 2 to accommodate the electroweak baryogenesis and the dark matter issues. The allowed DM mass in figure 1 is in the range $\mathrm{GeV} 60<m_{d}<320$ Gev for the $T_{c}^{+}$solution and in the range $\mathrm{GeV} 140<m_{d}<310 \mathrm{Gev}$ for the $T_{c}^{-}$solution. Therefore for both critical temperatures obtained in eq. (3.15) the pseudoscalar scenario respect the relic density and the first-order phase transition. The allowed DM mass for the scalar scenario is only in the range $\mathrm{GeV} 150<m_{d}<300 \mathrm{Gev}$ for the $T_{c}^{-}$solution which can easily be excluded by the future direct detection experiments such as XENON1T. This point has already been reported in [19]. For $T_{c}^{+}$solution there is no viable region in the scalar scenario.

Indirect detection bounds. The DM annihilation into SM particles is very suppressed after the thermal freeze-out in the early universe. Nevertheless, today in regions with high density of DM for instance in the Galactic Center (GC) the DM annihilation is probable. It is now well accepted from Fermi Large Area Telescope (Fermi-LAT) 6.5 years data that the gamma rays coming from the center of the galaxies are brighter for a few $\mathrm{GeV}$ than expected from other known sources [31]. The analyses of the GC gamma ray excess after considering different uncertainties puts an upper limit on the dark matter annihilation cross section in terms of the DM mass and the annihilation channel.

Here we examine our model against the Fermi-LAT bounds on DM annihilation cross section for two representative channels $b \bar{b}$ and $\tau^{+} \tau^{-}$which is relevant for DM masses up to $100 \mathrm{GeV}[31] .{ }^{3}$ For the current model we have computed the velocity-averaged annihilation

\footnotetext{
${ }^{3}$ Many thanks to Andrea Albert and Dmitry Malyshev for providing me with the Fermi-LAT data on figure 34 in [31].
} 


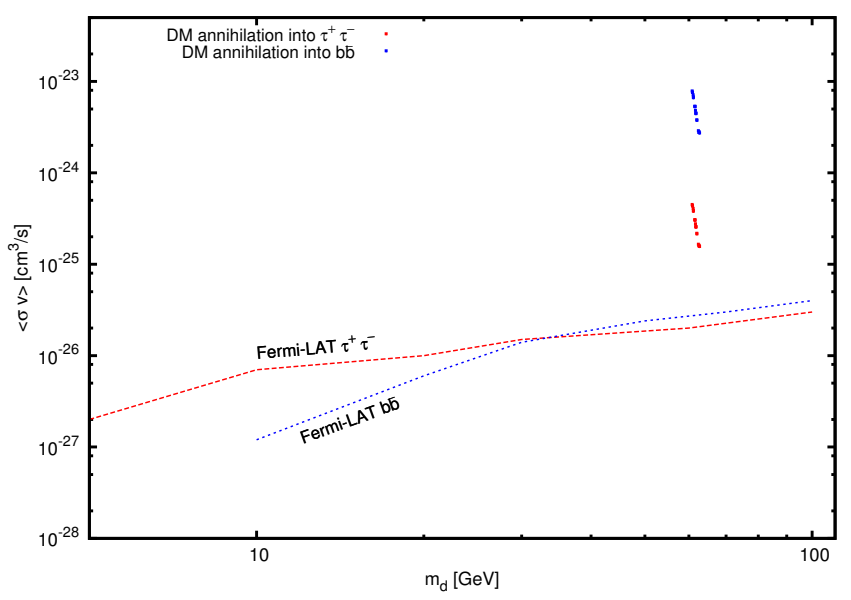

Figure 3. The dark matter velocity-averaged annihilation cross section into $b \bar{b}$ (blue) and $\tau^{+} \tau^{-}$ (red) for the DM mass up to $100 \mathrm{GeV}$. For both channels the DM masses $61-62 \mathrm{GeV}$ shown in figure 1 are excluded by the Fermi-LAT $b \bar{b}$ and $\tau^{+} \tau^{-}$upper limits.

cross section into two channels $b \bar{b}$ and $\tau^{+} \tau^{-}$for DM mass up to $100 \mathrm{GeV}$. All other constraints considered in the last sections are imposed in this computation. It is evident from figure 3 that for both channels the DM mass of $\sim 61-62 \mathrm{GeV}$ obtained before for the pseudoscalar mediator case in $T_{c}^{+}$solution (right plot in figure 1) is excluded by the FermiLAT limit. We have exploited the Fermi-LAT upper limit obtained from the generalized NFW dark matter density profile $(\gamma=1.25)$ in the center of the Galaxy.

After considering the Fermi-LAT constraint the viable DM mass with pseudoscalar mediator will be in the range $\sim 110-320 \mathrm{GeV}$ (see figure 1 ). Therefore the SM Higgs particle cannot decay into dark matter particle, hence the model is not bounded by invisible Higgs decay constraint. In figure 4 the DM mass is depicted against the pseudoscalar mediator mass considering all the aforementioned constraints. The points observed in this figure are as the following. First, for both $T_{c}^{+}$and $T_{c}^{-}$solutions the strongly first-order phase transition occurs in higher temperature for heavier dark matter particle. Second, the mass of the pseudoscalar mediator is in the range $290-620 \mathrm{GeV}$. Again the LHC exotic Higgs decay constraint which requires $m_{s}<m_{h} / 2$ is not applicable here.

The mono-jet searches at the LHC could also restrict DM models specially for low DM mass and heavier mediators where the dark matter production cross section becomes larger. It is shown in [32] that even for $m_{s}>2 m_{d}$ the LHC mono-jet search limit [33] does not constrain more the parameter space for the current model.

\section{Conclusion}

In this paper we have examined a fermionic dark matter model possessing a pseudoscalar or a scalar as the mediator whether it could explain the electroweak baryogenesis as well as evading the dark matter constraints. The (pseudo)scalar in the model is interpreted as a second Higgs-like particle beside the SM Higgs. We assumed that the scalars have zero vacuum expectation values in the symmetric phase but as the temperature comes 

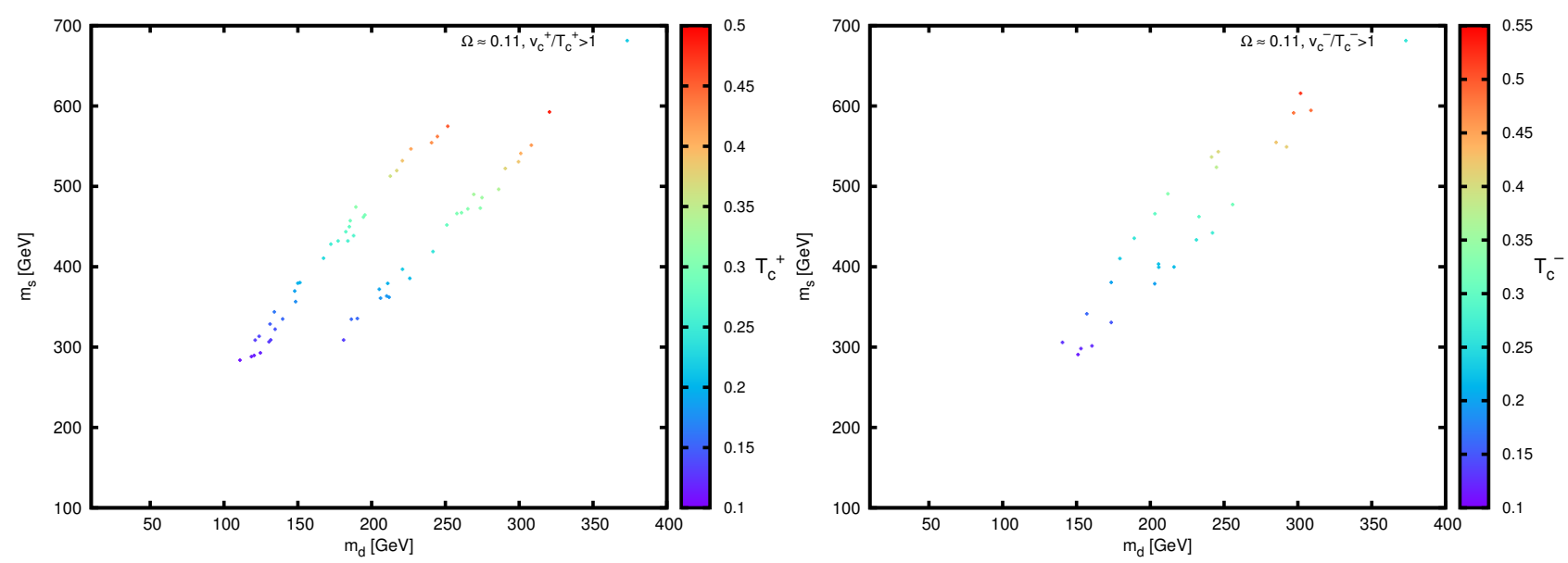

Figure 4. The pseudoscalar mediator mass against the allowed DM mass for left) $T_{c}^{+}$solution right) $T_{c}^{-}$solution. The critical temperature is shown in the color spectrum.

down with the expansion of the universe, the Higgs and the (pseudo)scalar undergo a nonzero vacuum expectation value and the electroweak phase transition takes place. We have obtained the critical temperature and the washout criterion analytically. Then we have computed the relic density using the micrOMEGAs package while we have considered all the constraints for the electroweak phase transition to be first-order. The numerical results show that there exist a viable space of parameters when the mediator is chosen to be a pseudoscalar with the dark matter mass in the range of $110-320 \mathrm{GeV}$ plus the resonance area with $m_{d}=61-62 \mathrm{GeV}$. If the SM-DM mediator in the theory is taken to be a scalar we have shown that there is only a small region that can satisfy all the constraints including the direct detection bound from XENON100/LUX and that will be excluded with the new bounds from the experiments such as XENON1T.

We then constrained more the model with the pseudoscalar mediator by the FermiLAT upper limit on DM annihilation into $b \bar{b}$ and $\tau^{+} \tau^{-}$which put a bound for DM mass up to $100 \mathrm{GeV}$. We have shown that this bound excludes the DM mass $61-62 \mathrm{GeV}$ we obtained before and the DM mass in the viable space becomes $m_{d}=110-320 \mathrm{GeV}$. The pseudoscalar mediator mass afterwards turned out to be $m_{s}=290-620 \mathrm{GeV}$, that means the model cannot be constrained more from exotic and invisible Higgs decay bounds at the LHC. It has also been pointed out that the LHC mono-jet search limit does not affect the viable space of parameters.

\section{A (Pseudo)scalar one-loop mass correction by dark matter fermion}

The first three terms in $\kappa_{h}$ in eq. (3.3) are the one-loop thermal correction in the SM sector, see [34]. The other terms in $\kappa_{h}$ and all the coefficients in $\kappa_{s}$ stem from the singlet (pseudo)scalar extension. For the details of the one-loop thermal calculations one may refer to [35]. The coefficients $\lambda$ and $\lambda_{s}$ in eq. (3.4) comes from the (pseudo)scalar mass correction with the Higgs or the (pseudo)scalar respectively in the loop. The signs of these two coefficients do not change for the scalar or the pseudoscalar. The third coefficient in 
eq. (3.4) is due to the (pseudo)scalar mass correction with the fermion dark matter in the loop. The sign of this term is however different for the scalar or the pseudoscalar. It will be plus for the scalar and minus for the pseudoscalar. We elaborate this point here.

The contribution from the fermion (dark matter) loop for the pseudoscalar mass correction is the following,

$$
\delta m_{(F-)}^{2}=g_{d}^{2} \int \frac{d^{4} k}{(2 \pi)^{4}} \frac{\operatorname{Tr}\left[\gamma^{5}\left(\gamma^{\mu} k_{\mu}+\gamma^{\mu} p_{\mu}+m_{d}\right) \gamma^{5}\left(\gamma^{\nu} k_{\nu}+m_{d}\right)\right]}{\left((k+p)^{2}-m_{d}^{2}\right)\left(k^{2}-m_{d}^{2}\right)},
$$

and the same correction for the scalar reads,

$$
\delta m_{(F+)}^{2}=g_{d}^{2} \int \frac{d^{4} k}{(2 \pi)^{4}} \frac{\operatorname{Tr}\left[\left(\gamma^{\mu} k_{\mu}+\gamma^{\mu} p_{\mu}+m_{d}\right)\left(\gamma^{\nu} k_{\nu}+m_{d}\right)\right]}{\left((k+p)^{2}-m_{d}^{2}\right)\left(k^{2}-m_{d}^{2}\right)} .
$$

Eqs. (A.1) and (A.2) can be written as,

$$
\delta m_{(F \pm)}^{2}=g_{d}^{2} \int \frac{d^{4} k}{(2 \pi)^{4}} \frac{ \pm 4 k^{2} \pm 4 p \cdot k+4 m_{d}^{2}}{\left((k+p)^{2}-m_{d}^{2}\right)\left(k^{2}-m_{d}^{2}\right)},
$$

where we have used the properties of the Dirac gamma matrices. The sign + stands for the scalar and the sign - stands for the pseudoscalar mass correction. At zero external momentum i.e. $p=0$ we have,

$$
\delta m_{(F \pm)}^{2}=g_{d}^{2} \int \frac{d^{4} k}{(2 \pi)^{4}} \frac{ \pm 4 k^{2}+4 m_{d}^{2}}{\left(k^{2}-m_{d}^{2}\right)^{2}} .
$$

Now in thermal field theory terminology the following substitutions provide us with the one-loop correction at finite temperature (see e.g. [36]),

$$
\begin{aligned}
k_{0} & \rightarrow 2 n i \pi T \quad \text { bosons } \\
k_{0} & \rightarrow(2 n+1) i \pi T \quad \text { fermions } \\
\int \frac{d^{4} k}{(2 \pi)^{4}} & \rightarrow T \sum_{n} \int \frac{d^{3} k}{(2 \pi)^{3}} .
\end{aligned}
$$

Using eqs. (A.4) and (A.5) we get,

$$
\begin{aligned}
& \delta m_{(F+)}^{2}=-4 T g_{d}^{2} \sum_{n=\text { odd }} \int \frac{d^{3} k}{(2 \pi)^{3}} \frac{1}{n^{2} \pi^{2} T^{2}+w_{k}^{2}}-\frac{2 m_{d}^{2}}{\left(n^{2} \pi^{2} T^{2}+w_{k}^{2}\right)^{2}}, \\
& \delta m_{(F-)}^{2}=+4 T g_{d}^{2} \sum_{n=\text { odd }} \int \frac{d^{3} k}{(2 \pi)^{3}} \frac{1}{n^{2} \pi^{2} T^{2}+w_{k}^{2}},
\end{aligned}
$$

where use has been made of $w_{k}^{2}=\vec{k}^{2}+m_{d}^{2}$. We see that the mass correction for the scalar contains a logarithmic divergence while for the pseudoscalar such a term does not exist.

Making the summations in eqs. (A.6) and (A.7) by

$$
\sum_{n=-\infty}^{+\infty} \frac{1}{n^{2}+x^{2}}=\frac{\pi}{x} \operatorname{coth} \pi x
$$


ignoring the second term in eq. (A.6) which does not contribute in $\mathcal{O}\left(T^{2}\right)$ we arrive at,

$$
\delta m_{(F \pm)}^{2}=\mp 2 g_{d}^{2} \int \frac{d^{3} k}{(2 \pi)^{3}} \frac{1}{w_{k}} \pm 4 T g_{d}^{2} \int \frac{d^{3} k}{(2 \pi)^{3}} \frac{1}{w_{k}} \frac{1}{1+e^{w_{k} / T}} .
$$

The first term in eq. (A.9) is the zero temperature contribution and the second term is the correction at finite temperature. At high temperature approximation, $m / T \ll 1$, this second term turns to be,

$$
\delta m_{(F \pm)}^{2}(T) \approx \pm \frac{1}{6} g_{d}^{2} T^{2}
$$

which accounts for the minus sign in eq. (3.4) for the pseudoscalar case and the plus sign for the scalar.

\section{Acknowledgments}

I would like to thank Karim Ghorbani, Alexander Pukhov and Nicolao Fornengo for very useful discussions. I am grateful for the hospitality and INFN-Torino university support when visiting the physics department of Torino university in February 2017 where this work was partially done.

Open Access. This article is distributed under the terms of the Creative Commons Attribution License (CC-BY 4.0), which permits any use, distribution and reproduction in any medium, provided the original author(s) and source are credited.

\section{References}

[1] S. Weinberg, Gauge and Global Symmetries at High Temperature, Phys. Rev. D 9 (1974) 3357 [INSPIRE].

[2] L. Dolan and R. Jackiw, Symmetry Behavior at Finite Temperature, Phys. Rev. D 9 (1974) 3320 [INSPIRE].

[3] A.D. Sakharov, Violation of CP Invariance, c Asymmetry and Baryon Asymmetry of the Universe, Pisma Zh. Eksp. Teor. Fiz. 5 (1967) 32 [INSPIRE].

[4] M.E. Shaposhnikov, Possible Appearance of the Baryon Asymmetry of the Universe in an Electroweak Theory, JETP Lett. 44 (1986) 465 [INSPIRE].

[5] ATLAS collaboration, Observation of a new particle in the search for the Standard Model Higgs boson with the ATLAS detector at the LHC, Phys. Lett. B 716 (2012) 1 [arXiv: 1207.7214] [INSPIRE].

[6] CMS collaboration, Observation of a new boson at a mass of $125 \mathrm{GeV}$ with the CMS experiment at the LHC, Phys. Lett. B 716 (2012) 30 [arXiv:1207.7235] [INSPIRE].

[7] Planck collaboration, R. Adam et al., Planck 2015 results. I. Overview of products and scientific results, Astron. Astrophys. 594 (2016) A1 [arXiv:1502.01582] [INSPIRE].

[8] WMAP collaboration, G. Hinshaw et al., Nine-Year Wilkinson Microwave Anisotropy Probe (WMAP) Observations: Cosmological Parameter Results, Astrophys. J. Suppl. 208 (2013) 19 [arXiv: 1212.5226] [INSPIRE]. 
[9] K. Ghorbani, Fermionic dark matter with pseudo-scalar Yukawa interaction, JCAP 01 (2015) 015 [arXiv: 1408.4929] [INSPIRE].

[10] K. Ghorbani, Renormalization group equation analysis of a pseudoscalar portal dark matter model, arXiv:1702.08711 [INSPIRE].

[11] P.-H. Gu, M. Lindner, U. Sarkar and X. Zhang, WIMP Dark Matter and Baryogenesis, Phys. Rev. D 83 (2011) 055008 [arXiv: 1009.2690] [INSPIRE].

[12] J.M. Cline and K. Kainulainen, Electroweak baryogenesis and dark matter from a singlet Higgs, JCAP 01 (2013) 012 [arXiv:1210.4196] [INSPIRE].

[13] M. Fairbairn and P. Grothaus, Baryogenesis and Dark Matter with Vector-like Fermions, JHEP 10 (2013) 176 [arXiv:1307.8011] [INSPIRE].

[14] M. Lewicki, T. Rindler-Daller and J.D. Wells, Enabling Electroweak Baryogenesis through Dark Matter, JHEP 06 (2016) 055 [arXiv: 1601.01681] [INSPIRE].

[15] M. Jiang, L. Bian, W. Huang and J. Shu, Impact of a complex singlet: Electroweak baryogenesis and dark matter, Phys. Rev. D 93 (2016) 065032 [arXiv:1502.07574] [INSPIRE].

[16] T.A. Chowdhury, M. Nemevšek, G. Senjanović and Y. Zhang, Dark Matter as the Trigger of Strong Electroweak Phase Transition, JCAP 02 (2012) 029 [arXiv:1110.5334] [INSPIRE].

[17] A. Menon, D.E. Morrissey and C.E.M. Wagner, Electroweak baryogenesis and dark matter in the NMSSM, Phys. Rev. D 70 (2004) 035005 [hep-ph/0404184] [INSPIRE].

[18] V. Barger, P. Langacker, M. McCaskey, M. Ramsey-Musolf and G. Shaughnessy, Complex Singlet Extension of the Standard Model, Phys. Rev. D 79 (2009) 015018 [arXiv:0811. 0393] [INSPIRE].

[19] T. Li and Y.-F. Zhou, Strongly first order phase transition in the singlet fermionic dark matter model after LUX, JHEP 07 (2014) 006 [arXiv: 1402.3087] [INSPIRE].

[20] A. Addazi and A. Marciano, Gravitational waves from dark first order phase transitions and dark photons, arXiv:1703.03248 [INSPIRE].

[21] S.R. Coleman and E.J. Weinberg, Radiative Corrections as the Origin of Spontaneous Symmetry Breaking, Phys. Rev. D 7 (1973) 1888 [INSPIRE].

[22] S. Esch, M. Klasen and C.E. Yaguna, Detection prospects of singlet fermionic dark matter, Phys. Rev. D 88 (2013) 075017 [arXiv: 1308.0951] [inSPIRE].

[23] L. Lopez-Honorez, T. Schwetz and J. Zupan, Higgs portal, fermionic dark matter and a Standard Model like Higgs at 125 GeV, Phys. Lett. B 716 (2012) 179 [arXiv:1203.2064] [INSPIRE].

[24] M. Pospelov and A. Ritz, Higgs decays to dark matter: beyond the minimal model, Phys. Rev. D 84 (2011) 113001 [arXiv:1109.4872] [INSPIRE].

[25] LUX collaboration, D.S. Akerib et al., Results from a search for dark matter in the complete LUX exposure, Phys. Rev. Lett. 118 (2017) 021303 [arXiv: 1608.07648] [InSPIRE].

[26] XENON100 collaboration, E. Aprile et al., XENON100 Dark Matter Results from a Combination of 477 Live Days, Phys. Rev. D 94 (2016) 122001 [arXiv:1609.06154] [INSPIRE].

[27] XENON collaboration, E. Aprile et al., First Dark Matter Search Results from the XENON1T Experiment, arXiv:1705.06655 [INSPIRE]. 
[28] G. Bélanger, F. Boudjema, A. Pukhov and A. Semenov, MicrOMEGAs4.1: two dark matter candidates, Comput. Phys. Commun. 192 (2015) 322 [arXiv:1407.6129] [INSPIRE].

[29] D. Bödeker and G.D. Moore, Can electroweak bubble walls run away?, JCAP 05 (2009) 009 [arXiv:0903.4099] [INSPIRE].

[30] J. Kozaczuk, Bubble Expansion and the Viability of Singlet-Driven Electroweak Baryogenesis, JHEP 10 (2015) 135 [arXiv: 1506.04741] [INSPIRE].

[31] Fermi-LAT collaboration, M. Ackermann et al., The Fermi Galactic Center GeV Excess and Implications for Dark Matter, Astrophys. J. 840 (2017) 43 [arXiv:1704.03910] [INSPIRE].

[32] S. Baek, P. Ko and J. Li, Minimal renormalizable simplified dark matter model with a pseudoscalar mediator, Phys. Rev. D 95 (2017) 075011 [arXiv:1701.04131] [INSPIRE].

[33] ATLAS collaboration, Search for new phenomena in final states with an energetic jet and large missing transverse momentum in pp collisions at $\sqrt{s}=13$ TeV using the ATLAS detector, Phys. Rev. D 94 (2016) 032005 [arXiv: 1604.07773] [INSPIRE].

[34] M.E. Carrington, The effective potential at finite temperature in the Standard Model, Phys. Rev. D 45 (1992) 2933 [INSPIRE].

[35] T.A. Chowdhury, A Possible Link between the Electroweak Phase Transition and the Dark Matter of the Universe, Ph.D. Thesis, SISSA, Trieste, Italy (2014).

[36] A. Das, Finite Temperature Field Theory, World scientific Lecture Notes In Physics, World Scientific, (1997). 